Archived version from NCDOCKS Institutional Repository http://libres.uncg.edu/ir/asu/

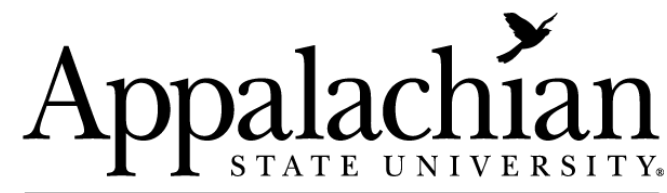

B O O N E, NORT H C A R O L I A

\section{Limitations And Benefits Of ARISA Intra-Genomic Diversity Fingerprinting}

\author{
By: Radu Popa, Rodica Popa, Matthew J. Marshall, Hien Nguyen, \\ Bradley M. Tebo, \& Suzanna Brauer
}

\begin{abstract}
Monitoring diversity changes and contamination in mixed cultures and simple microcosms is challenged by fast community structure dynamics, and the need for means allowing fast, cost-efficient and accurate identification of microorganisms at high phylogenetic resolution. The method we explored is a variant of Automated rRNA Intergenic Spacer Analysis based on Intra-Genomic Diversity Fingerprinting (ARISA-IGDF), and identifies phylotypes with multiple 16S-23S rRNA gene Intergenic Transcribed Spacers. We verified the effect of PCR conditions (annealing temperature, duration of final extension, number of cycles, group-specific primers and formamide) on ARISA-IGD fingerprints of 44 strains of Shewanella. We present a digitization algorithm and data analysis procedures needed to determine confidence in strain identification. Though using stringent PCR conditions and group-specific primers allow reasonably accurate identification of strains with three ARISA-IGD amplicons within the 82-1000 bp size range, ARISA-IGDF is best forphylotypes with $\geq 4$ unambiguously different amplicons. This method allows monitoring the occurrence of culturable microbes and can be implemented in applications requiring high phylogenetic resolution, reproducibility, low cost and high throughput such as identifying contamination and monitoring the evolution of diversity in mixed cultures and low diversity microcosms and periodic screening of small microbial culture libraries.
\end{abstract}

Popa, Radu \& Rodica, Marshall, M., Nguyen, H., Tebo, M., \& Braver, S. (2009). Limitations and Benefits of ARISA Intra-genomic Diversity Fingerprinting. Journal of Microbiological Methods. Volume 78, Issue 2, 2009. Pages 111 -1 18. https://doi.org/10.1016/i.mimet.2009.06.005. Publisher version of record available at: http://www.sciencedirect.com/science/article/pii/S0167701209001821 


\title{
Limitations and benefits of ARISA intra-genomic diversity fingerprinting
}

\author{
Radu Popa ${ }^{\mathrm{a}, *}$, Rodica Popa ${ }^{\mathrm{a}}$, Matthew J. Mashall ${ }^{\mathrm{b}}$, Hien Nguyen ${ }^{\mathrm{a}}$, Bradley M. Tebo ${ }^{\mathrm{c}}$, Suzanna Brauer ${ }^{\mathrm{c}, 1}$ \\ a Department of Biology, Portland State University, 1719 SW 10th Ave., SB2 Room 246, Portland, OR, 97201, USA \\ ${ }^{\mathrm{b}}$ Biological Sciences Division, Pacific Northwest National Laboratory, Richland, WA, 99352, USA \\ c Department of Environmental and Biomolecular Sciences, Oregon Health and Science University, 20000 NW Walker Rd., Beaverton, OR, 97006, USA
}

Keywords:

ARISA-IGDF

ITS

Molecular fingerprinting

qPCR

\begin{abstract}
A B S T R A C T
Monitoring diversity changes and contamination in mixed cultures and simple microcosms is challenged by fast community structure dynamics, and the need for means allowing fast, cost-efficient and accurate identification of microorganisms at high phylogenetic resolution. The method we explored is a variant of Automated rRNA Intergenic Spacer Analysis based on Intra-Genomic Diversity Fingerprinting (ARISAIGDF), and identifies phylotypes with multiple 16S-23S rRNA gene Intergenic Transcribed Spacers. We verified the effect of PCR conditions (annealing temperature, duration of final extension, number of cycles, group-specific primers and formamide) on ARISA-IGD fingerprints of 44 strains of Shewanella. We present a digitization algorithm and data analysis procedures needed to determine confidence in strain identification. Though using stringent PCR conditions and group-specific primers allow reasonably accurate identification of strains with three ARISA-IGD amplicons within the $82-1000$ bp size range, ARISA-IGDF is best for phylotypes with $\geq 4$ unambiguously different amplicons. This method allows monitoring the occurrence of culturable microbes and can be implemented in applications requiring high phylogenetic resolution, reproducibility, low cost and high throughput such as identifying contamination and monitoring the evolution of diversity in mixed cultures and low diversity microcosms and periodic screening of small microbial culture libraries.
\end{abstract}

\section{Contents}

1. Introduction . . . 111

2. Materials and methods . . . . . . . . . . . . . . . . . . . . . . . . . . . . . . . . . . . . . . . 112

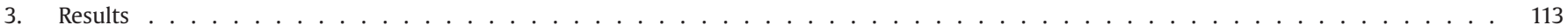

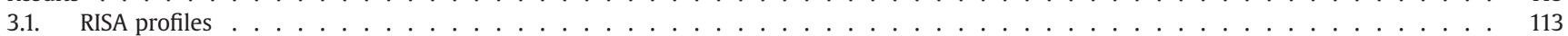

3.2. Optimizing the PCR conditions . . . . . . . . . . . . . . . . . . . . . . . . . . . . . . . . . . . . 113

3.3. Constructing fingerprints .. . . . . . . . . . . . . . . . . . . . . . . . . . . . . . . . . . 115

3.4. Confidence in phylotype identification . . . . . . . . . . . . . . . . . . . . . . . . . . . . . . . . . . . . . . . . . . . 116

4. Discussions ... . . . . . . . . . . . . . . . . . . . . . . . . . . . . . . . . . . . 117

5. Conclusions . . . . . . . . . . . . . . . . . . . . . . . . . . . . . . . . . . . . . . . . . . . . . . . 117

Acknowledgements . . . . . . . . . . . . . . . . . . . . . . . . . . . . . . . . . . . . 118

References . . . . . . . . . . . . . . . . . . . . . . . . . . . . . . . . . . . . . . . . . . . . 118

\section{Introduction}

Molecular approaches that aim to monitor changes in community structure in mixed cultures and low diversity microcosms are

\footnotetext{
* Corresponding author. Tel.: +1 503725 9503; fax: +1503 7253888. E-mail address: rpopa@pdx.edu (R. Popa).
}

1 Present address: Department of Biology, Appalachian State University, 572 Rivers Street, Boone, NC 28608, USA. challenged by considerations related to cost, small $16 \mathrm{~S}$ rRNA gene sequence differences between otherwise distinct phylotypes, limited phylogenetic resolution of some fingerprinting methods, and potentially large changes in community structure over time. For monitoring mixed communities at subspecies-level resolution cloning/sequencing and metagenomic sequencing are impractical, and fingerprinting methods are sought that are fast, low cost and offer high phylogenetic resolution. Automated rRNA Intergenic Spacer Analysis (ARISA) is a high resolution repetitive PCR fingerprinting method based on the size variability of the 
16S-23S Intergenic Transcribed Spacers (ITSs) (Garcia-Martinez et al., 1999). To date, ARISA, and its simpler variant RISA, were applied to studying overall changes in diversity and to some lesser extent for microbial identification (Bergamo et al., 2004; Grassi et al., 2006; Heuer et al., 1997; Hewson et al., 2003; Jensen et al., 1993; Ni et al., 2007; Siering and Ghiorse, 1996; Torzilli et al., 2006). A number of modifications implemented in ARISA (such as qPCR, sequencing, group-specific primers, fluorescent densitometry, and statistical means to describe diversity and similarity) led to sharp electrophoretic peaks, high throughput, and high phylogenetic resolution (Acinas et al., 1999; Fisher and Triplett, 1999; Hewson et al., 2003; Offre et al., 2007; Ranjard et al., 2003; Robleto et al., 1998). Still, objective technical constrains restrict the usefulness of this method in very complex natural samples (Hewson and Fuhrman, 2006; Loisel et al., 2006). The most notable limitations of ARISA are: use of taxonomically-ambiguous entities (i.e. Operational Taxonomic Units or OTUs); (b) hard to describe fingerprints of uncultured microbes; (c) numerous peaks when "universal primers" are used on complex samples; and (d) uneven confidence in identification across the phylogenetic spectrum. Because the concept of species remains ambiguous in Bacteria, and because more work is needed to define ARISA's phylogenetic resolution, we prefer using the term phylotype (rather than species, strain or OTU) to discuss the entities identified by this method. By extension, phylogroup represents a group of phylotypes revealed by a fingerprinting approach (e.g. specific PCR conditions, specific primers, specific numerical analysis for identifying amplicons, etc.). In this study the genus Shewanella was used as a model phylogroup, and the different strains of Shewanella showing distinct ARISA fingerprints are defined as phylotypes.

Our hypothesis was that an ARISA-based protocol can be developed that will allow fast, high throughput and low cost identification of microbial phylotypes from the Shewanella phylogroup at species and subspecies-level resolution. The variant of ARISA we explored identifies phylotypes based on the Intra-Genomic Diversity (IGD) of ITSs, hence IGD Fingerprinting (IGDF). We studied requirements, limitations and benefits of this approach and verified the effect of PCR conditions (i.e. different pairs of primers, duration of the final PCR extension, annealing temperature, number of PCR cycles and presence of formamide) on the PCR amplification yield, the formation of "ghost" peaks, resolution, peak shape and fingerprint reproducibility. We measured errors in estimating the size of amplicons and present an algorithm for quantifying the confidence in strain identification. We introduce a digitization protocol for ARISA-IGDF libraries that will facilitate the automation of fingerprint comparison.

\section{Materials and methods}

The genus Shewanella was selected because the genomes of the Shewanella strains sequenced to date revealed sufficiently high diversity of intra-genomic ITSs, and because Shewanella-specific 16S rRNA gene primers exist (Todorova and Costello, 2006). A total number of 44 Shewanella strains were obtained from four culture collections (Table 1). Genomic DNA was obtained by direct heat extraction from cell pellets (modified after: Cook and Meyers, 2003; Emerson and Moyer, 1997; Mazza et al., 2003; Siering and Ghiorse, 1996). After 24-48 h of growth in liquid LB medium in aerobic conditions cells were centrifuged ( $2 \mathrm{~min} ; 7500 \mathrm{~g} ; 20^{\circ} \mathrm{C}$ ), and washed twice in $500 \mu \mathrm{l}$ TE buffer ( $10 \mathrm{mM}$ Tris $\mathrm{HCl} ; 1 \mathrm{mM}$ EDTA; pH 8). After vortexing for 2 min with $2 \mathrm{~mm}$ glass beads the genomic DNA was extracted by heating for $10 \mathrm{~min}$ on a $100{ }^{\circ} \mathrm{C}$ water bath. The cellular debris was pelleted by centrifugation $\left(3 \mathrm{~min} ; 7500 \mathrm{~g} ; 20^{\circ} \mathrm{C}\right.$ ) and $\sim 70-$ $80 \%$ of the supernatant was recovered and used to quantify the dsDNA using the PicoGreen method (Molecular Probes, CA).

The DNA extracts were diluted to $1 \mu \mathrm{g} / \mathrm{ml}$ in $\mathrm{ddH}_{2} \mathrm{O}$ and $3.6 \mu \mathrm{l}$ of each template solution was used in $10 \mu \mathrm{l}$ PCR reactions (Fermentas Inc., MD) with $0.4 \mu \mathrm{l}$ of each $10 \mu \mathrm{M}$ primer. We used the primers: ITSF (5'GTCGTAACAAGGTAGCCGTA); ITSReub (5'GCCAAGGCATCCACC)
Table 1

Strains used in this study (nomenclature after Fredrickson et al., 2008).

\begin{tabular}{|c|c|c|c|c|c|}
\hline A & B & $\mathrm{C}$ & A & B & \\
\hline 1 & Shewanella oneidensis MR1 & $\mathrm{a}$ & 27 & Shewanella sp. HRCR1(1A) & \\
\hline 2 & Shewanella amazonensis SB2B & a & 28 & Shewanella sp. HRCR2(1B) & \\
\hline 3 & Shewanella baltica OS155 & $\mathrm{a}$ & 29 & Shewanella sp. HRCR3(1C) & \\
\hline 4 & Shewanella frigidimarina NCIMB400' & $\mathrm{a}$ & 30 & Shewanella sp. HRCR4(2A) & \\
\hline 5 & Shewanella denitrificans OS217 & $\mathrm{a}$ & 31 & Shewanella sp. HRCR5(2B) & \\
\hline 6 & Shewanella loihica PV4 & $\mathrm{a}$ & 32 & Shewanella sp. HRCR5(2B) & \\
\hline 7 & Shewanella putrefaciens $200^{\prime}$ & $\mathrm{a}$ & 33 & Shewanella sp. HRCR7(3A) & \\
\hline 8 & Shewanella putrefaciens CN32 & a & 34 & Shewanella sp. $H R R 8(3 B)$ & \\
\hline 9 & Shewanella sp. MR7 & $\mathrm{a}$ & 35 & Shewanella sp. $H R C R 9(3 C)$ & \\
\hline 10 & Shewanella sp. PV4 & $\mathrm{a}$ & 36 & Shewanella sp. HRCR10(4A) & \\
\hline 11 & Shewanella frigidimarina NCIMB400" & $\mathrm{a}$ & 37 & Shewanella sp. HRCR11(4B) & \\
\hline 12 & Shewanella baltica OS195 & $\mathrm{a}$ & 38 & Shewanella sp. HRCR12(4C) & \\
\hline 13 & Shewanella sp. MR4 & $\mathrm{a}$ & 39 & Shewanella sp. HRCR13(5A) & \\
\hline 14 & Shewanella sp. L1250 & $\mathrm{a}$ & 40 & Shewanella sp. HRCR14(5B) & \\
\hline 15 & Shewanella putrefaciens $200^{\prime \prime}$ & $\mathrm{a}$ & 41 & Shewanella sp. HRCR15(5C) & \\
\hline 16 & Shewanella sp. W3-18-1 & a & 42 & Shewanella sp. HRCR18(4D) & \\
\hline 17 & Shewanella sp. ANA-3 & $\mathrm{a}$ & 43 & Shewanella sp. HRCR19(5D) & \\
\hline 21 & Shewanella sp. HRCR21 & $\mathrm{b}$ & 44 & Shewanella sp. HRCR20(5E) & \\
\hline 22 & Shewanella sp. HRCR22 & $\mathrm{b}$ & 45 & Shewanella sp. OGI(A) & \\
\hline 23 & Shewanella sp. HRCR23 & $\mathrm{b}$ & 46 & Shewanella sp. OGI45 & \\
\hline 24 & Shewanella sp. HRCR24 & $\mathrm{b}$ & 47 & Shewanella sp. OGI & \\
\hline 25 & Shewanella sp. HRCR25 & $\mathrm{b}$ & 48 & Shewanella sp. OGI 104 & \\
\hline 26 & Shewanella sp. HRCR26 & $\mathrm{b}$ & 50 & E. coli CFT073 & \\
\hline
\end{tabular}

(A) Strain number in the PSU clone library. (B) Species and strain according to the source culture collection. (C) Source: (a) University of Southern California; (b) Pacific Northwest National Laboratory; (c) OGI School of Science and Engineering at Oregon Health and Sciences University; (d) ATCC. Strains \# 34 and 50 do not belong to the genus Shewanella and were used as controls.

and Shew1259F (5'TACAGAGGGTTGCAAAGCC). The ITSF and ITSReub primers were proposed earlier as "bacterial universal" RISA primers (Cardinale et al., 2004) (3). Shew1259F is a putative "Shewanellaspecific" primer redesigned after Todorova and Costello (2006). ITSReub was fluorescently labeled with Cy3, FAM or HEX; HEX has the advantage of non-overlapping fluorescence with SYBR green (used during qPCR amplifications), or with ROX (present in the ladder used for electrophoretic separation). Non-labeled primers were obtained from IDT DNA (Integrated DNA Technologies, IA); fluorescentlylabeled primers were obtained from Operon Biotechnologies (AL). Formamide (Sigma, F9037) was used at a final concentration of $1.5 \%$. The following range of PCR conditions was tested: denaturation ( $15 \mathrm{~min}$ at $\left.95{ }^{\circ} \mathrm{C}\right) ; 35$ to 50 cycles of amplification $\left(30 \mathrm{~s}\right.$ at $94{ }^{\circ} \mathrm{C} ; 30 \mathrm{~s}$ annealing between $45{ }^{\circ} \mathrm{C}$ and $65{ }^{\circ} \mathrm{C}$; and $1 \mathrm{~min}$ at $72{ }^{\circ} \mathrm{C}$ ); and final extension (between 0 and $59 \mathrm{~min}$ at $72{ }^{\circ} \mathrm{C}$ ). For common amplifications we used a GeneAmp 2400 thermocycler (Perkin Elmer) and a Fermentas PCR kit (Fermentas, CA); for temperature gradient PCR we used a Veriti Thermal Cycler (Applied Biosystems, CA) and the Fermentas PCR kit; for qPCR reactions we used a Stratagene MX3005P instrument and a SYBR Green PCR kit (QIAGEN, CA).

All PCR amplifications were separated by electrophoresis in $0.7 \%$ agarose/TAE gels (100V; 20 min), stained with ethidium bromide and visualized at $302 \mathrm{~nm}$. For RISA profiles via polyacrylamide gel electrophoresis (PAGE) we used 5\% polyacrylamide gels with 44:1 acrylamide:bis molar ratio ( $100 \mathrm{~V} ; 5 \mathrm{C} ; 11 \mathrm{~h}$ ), visualization by laser scanning fluorescence densitometry on a Typhoon 9200 Variable Mode gel imager using the program Typhoon scan; the images were analyzed with ImageQuant and adjusted for contrast, brightness and intensity.

For obtaining ARISA profiles $1 \mu$ of each PCR product (or dilutions of it) was mixed with $12.1 \mu \mathrm{l} \mathrm{HiDi}$ formamide and $0.4 \mu \mathrm{l}$ ROX1000 ladder (Applied Biosystems, CA), heat-denatured (5 min; $95{ }^{\circ} \mathrm{C}$ ), cooled fast at $-40{ }^{\circ} \mathrm{C}$ and injected in triplicates in a ABI 310 capillary sequencer with POP4 resin (Applied Biosystems, CA). Controls were also used based on different replicates and dilutions of ladder and $E$. coli amplificates. The electrophoretic separation occurred at $15 \mathrm{kV}$. Peak resolution decreased with fragment size and became $>0.28 \mathrm{bp}$ 


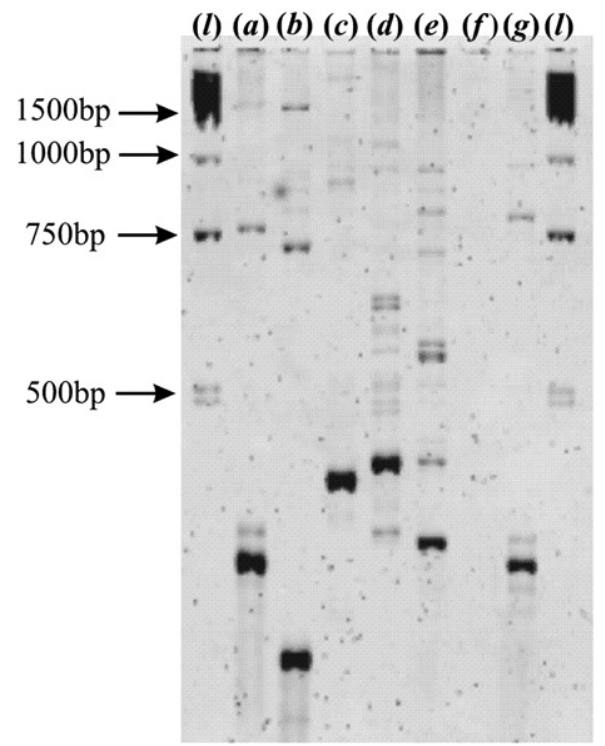

Fig. 1. Electropherogram of RISA profiles of isolated strains. This analysis was used to verify a potential mislabeling in the Shewanella library. For PCR amplification we used ITSF/Cy3-ITSReub primers and $63{ }^{\circ} \mathrm{C}$ annealing temperature. Except for the lane (f) where 1:10 dilutions were used, all lanes were loaded with $5 \mu \mathrm{l}$ of PCR products. (a) S. oneidensis MR1. (b) S. amazonensis SB2B. (c) S. baltica OS155. (d) S. putrefaciens 200. (e) S. putrefaciens CN32. (f) S. putrefaciens CN32 and (g) E. coli CFT073. (1) Ladder.

above $500 \mathrm{bp}$; yet, because in some Shewanella strains some important diagnostic peaks are $>500$ bp long we analyzed peaks as large as $1000 \mathrm{bp}$. The electropherograms were downloaded in GeneScan v2.5 (ABI), analyzed with Peak Scanner v1.0 (ABI) to obtain fragment sizes, range and fluorescence, and the numerical data was processed in Microsoft Excel. We used the following cutoffs: (1) eliminated all peaks smaller than 82 bp (i.e. the sum between the size of the primers and the remaining parts of the 16S rRNA and 23S rRNA genes); (2) eliminated all peaks larger than $1000 \mathrm{bp}$; (3) eliminated all peaks with fluorescence $<5$ times the background (Hewson et al., 2003); and (4) eliminated all peaks with a fluorescence $<0.1 \%$ from the total fluorescence of all larger peaks; and (5) analyzed $\leq 20$ largest peaks for each strain. All PCR amplifications were produced in at least triplicates, and each PCR product was read in at least two dilutions.
Strain-diagnostic peaks were identified based on co-occurrence across replicates. For a specific peak to be validated as diagnostic it had to be present in $100 \%$ of $\leq 4$ replicates, or $\geq 80 \%$ of $\geq 5$ replicates. To determine the fluorescence of peaks saturating the detector we repeated electropherograms using diluted samples. Verifying if peaks from different electropherograms represent the same amplicons required determining the relationship between fragment size (in bp) and Standard Deviation (SD) of fragment size (Eq. (1)), and the relationship between peak abundance and the $S D$ of abundance (Eq. (2)).

$S D$ of fragment size $($ in bp $)=0.000315 \cdot$ Fragment size $($ in bp $)+0.126$

(based on five replicate readings of one sample of $S$. amazonensis SB2B)

SD of peak abundance (in \%) $=0.0684 \cdot$ Peak abundance $($ in $\%)+0.934$

(based on five independent PCR amplifications of $S$. amazonensis SB2B)

Two peaks, $x$ and $y$, from two electropherograms were considered putatively dissimilar at $97 \%$ confidence, if the $x+/-S D_{x}$ range did not overlap the $y+/-S D_{y}$ range.

\section{Results}

\subsection{RISA profiles}

All strains were characterized first by RISA profiling using PAGE. Though this method is less precise than ARISA and adds ghost bands due to heteroduplexes and homoduplexes (Daffonchio et al., 2003), it has the advantage of being fast, allowing large scale and cost effective screening, identifies cross-contamination and mislabeled strains in microbial libraries (Fig. 1), and helps in formulating dilution series for sequencer-based fingerprinting.

\subsection{Optimizing the PCR conditions}

Increasing the duration of the final $72{ }^{\circ} \mathrm{C}$ extension in PCR reactions helps in minimizing peak shoulders caused by TAQ polymeraseinduced artifacts (Torzilli et al., 2006). Expecting that too short final





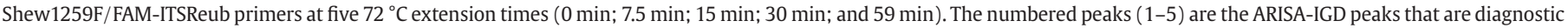

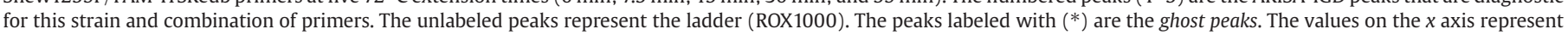
sizes (in bp) for some of the ladder peaks. Electropherograms were stretched vertically for peak \#3 to reach $100 \%$ and thus allow image comparisons. 


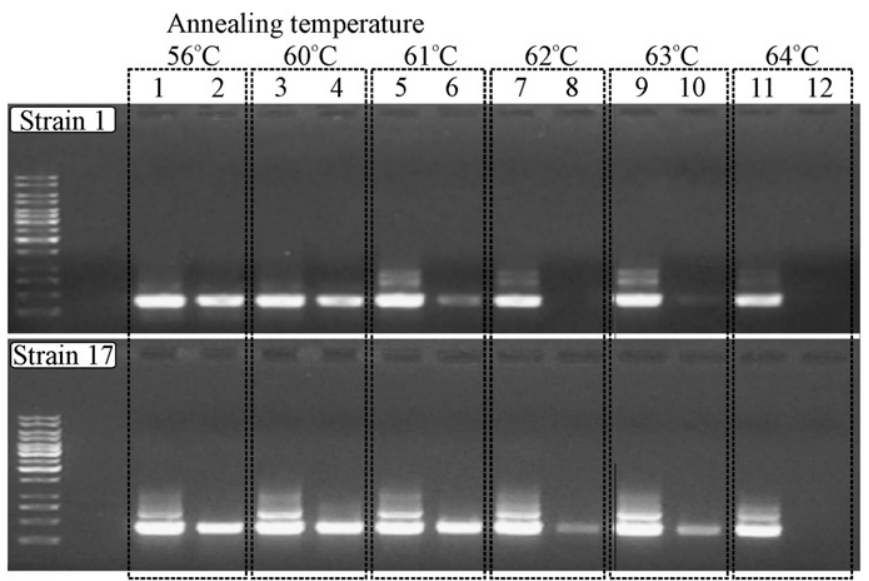

Fig. 3. The effect of annealing temperature and $1.5 \%$ formamide on the PCR amplification in S. oneidensis MR1 (Strain \#1) and Shewanella sp. ANA (Strain \#17). The ladder, at the far left, was $1 \mathrm{~kb}$ IDNA (Fermentas). The sample gel load was $5 \mu \mathrm{l}$ of PCR products. Odd numbers represent the no-formamide treatments; even numbers represent the formamide treatments. The electropherograms were obtained on $0.7 \%$ agarose/TAE gels, (120 V; $30 \mathrm{~min})$, stained with ethidium bromide and visualized at $302 \mathrm{~nm}$.

extension will produce false positives (i.e. "ghost" peaks), we verified the effect of five extension times on ARISA-IGDF patterns on DNA from S. amazonensis SB2B (Fig. 2, Q2404, p45). We found no significant differences in peak shape between the different extension times (results not shown), and that ghost peaks existed in all $<30 \mathrm{~min}$ extension treatments. A final extension of $45 \mathrm{~min}$ at $72{ }^{\circ} \mathrm{C}$ was used in all subsequent PCR amplifications.

Formamide was reported to increase primer specificity during PCR reactions (Grassi et al., 2006; Sarkar et al., 1990; Zhang and Albert, 1996), and thus may help in implementing group-specific primers in ARISA-IGD fingerprinting. However, formamide may also inhibit the PCR reaction when appropriate primers are used on target phylogroups. We verified the effect of $1.5 \%$ formamide on the maximum annealing temperature on eight strains of Shewanella (\#1, \#2, \#3, \#7, \#8, \#9, \#11 and \#17; see: Table 1) using the primers Shew1259F/ ITSReub (two examples are given in Fig. 3). Based on these eight strains the best annealing temperature for Shewanella was $64{ }^{\circ} \mathrm{C}$, and dropped by $\sim 3-4^{\circ}$ when formamide was used. Peak patterns (mainly relative abundances of different peaks) were also affected by formamide (results not shown). To verify the effect of formamide on the overall efficiency of amplification with the primers Shew1259F/

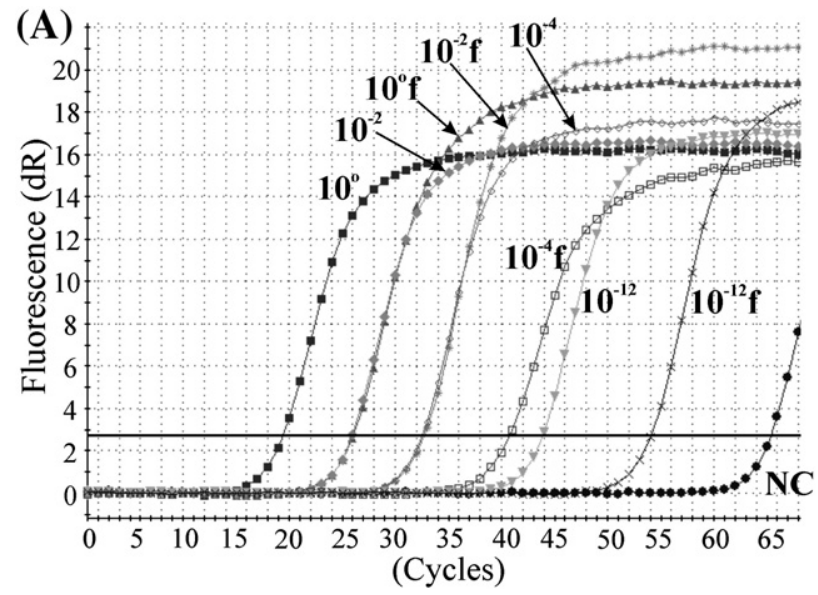

(A) qPCR of E.coli CFT073

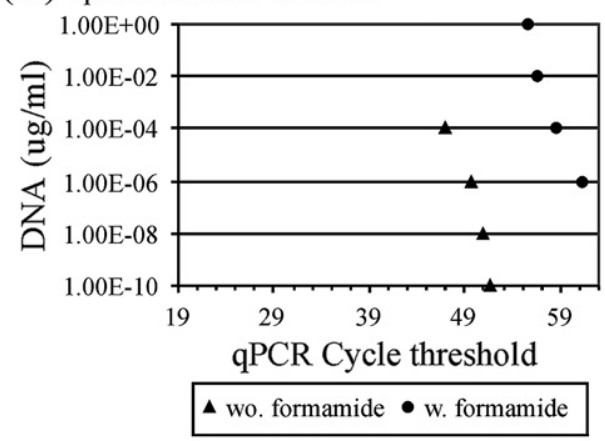

(B) qPCR of Shewanella sp. ANA

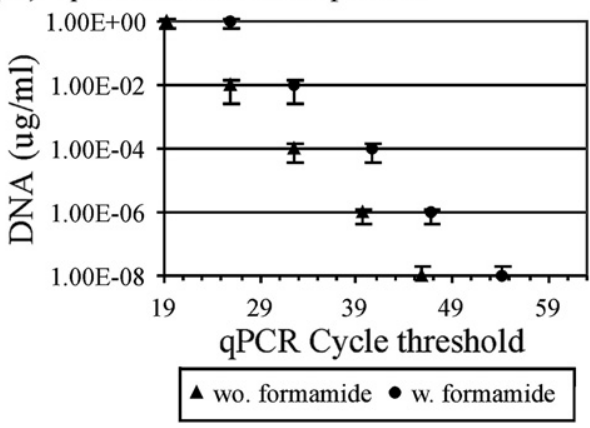

Fig. 5. The efficiency of qPCR amplification of genomic DNA from Shewanella sp. ANA-3 with the primers Shew1259F/HEX-ITSReub, at $60{ }^{\circ} \mathrm{C}$ annealing temperature with and without $1.5 \%$ formamide. The $y$ axis indicates the concentration of DNA in template solutions. Error bars are 1SD of DNA concentrations calculated from the variance of the cycle threshold $(C t)$.

ITSReub we produced serial dilutions ( $10^{\circ}$ to $10^{-12}$ of genomic DNA; where $10^{\circ}=1 \mu \mathrm{g} \mathrm{DNA} \mathrm{ml}{ }^{-1}$ in the template solutions), and amplified by qPCR. All qPCR amplifications were done in triplicate. Formamide inhibited the amplification of Shewanella DNA about 100 times (Figs. 4A and 5), with no notable changes in ARISA patterns and variability (discussed below). Although Shew $1259 \mathrm{~F}$ is supposed to be "Shewanella-specific" and the annealing temperature was high $\left(60{ }^{\circ} \mathrm{C}\right)$, amplification occurred eventually in a control strain of $E$. coli as well if enough cycles were applied (Fig. 4B). This may partly be due to using the "bacterial universal" ARISA reverse primer (ITSReub), which lowers the stringency of amplification. Overall, the efficiency of amplifying $E$. coli DNA under these conditions was very low $\left(<10^{-7}\right)$ relative to Shewanella, the variability of the cycle threshold $(C t)$ in $E$.



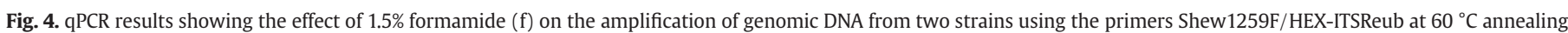

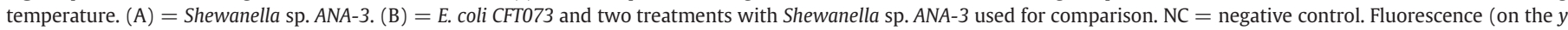

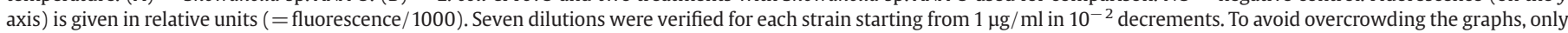
a few relevant amplification plots are shown. 


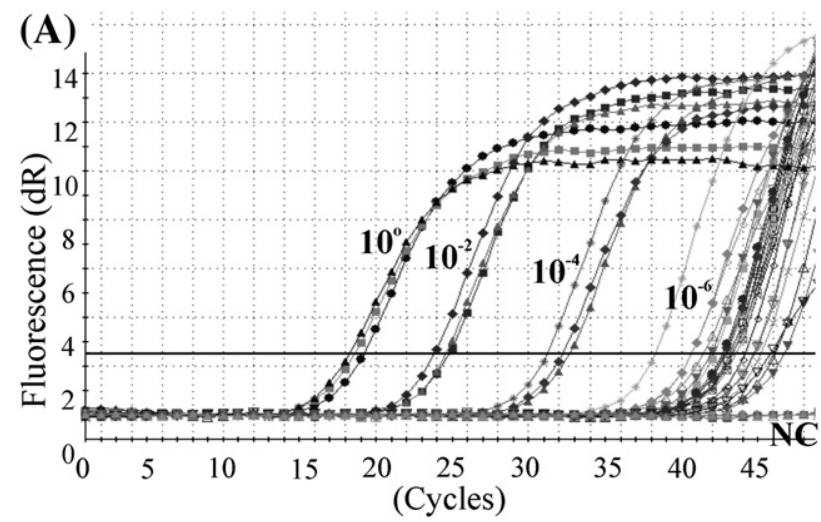

(B)

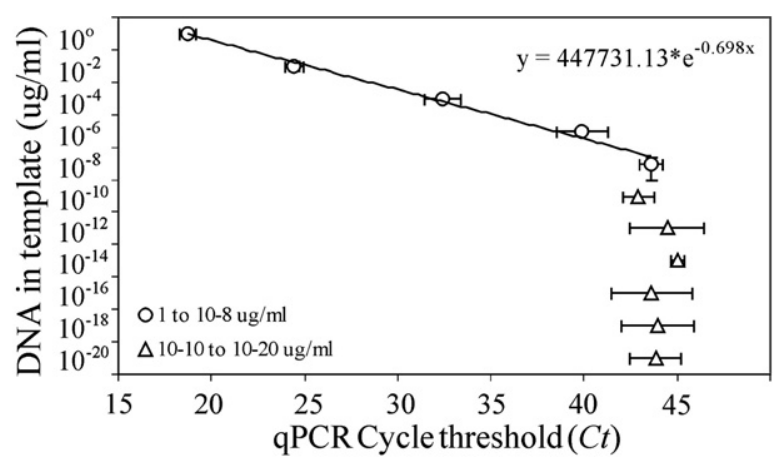

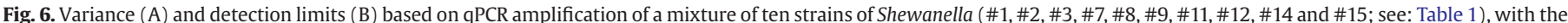

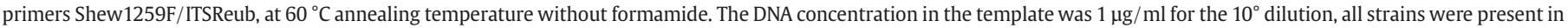

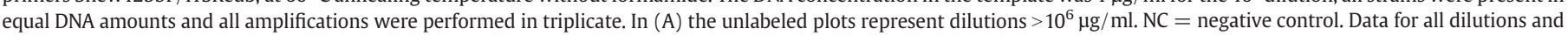

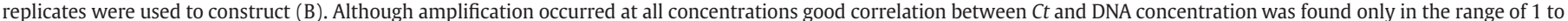
$10^{-8} \mu \mathrm{g} / \mathrm{ml}$ DNA. The error bars in (B) represent $1 S D$ of $C t$.

coli amplifications was very large (results not shown) and the DNA concentration vs. Ct dependency was steep. Therefore the incertitude when quantifying the concentration of $E$. coli DNA from $C t$ remains large. Formamide had a stronger inhibitory effect on the amplification of the control E. coli DNA than on Shewanella DNA. Based on these results, all PCR amplifications used to construct the Shewanella ARISAIGDF library were made at $60{ }^{\circ} \mathrm{C}$ annealing without formamide.

To define the relationship between the concentration of DNA template and the optimal number of PCR cycles in ARISA-IGDF, we used qPCR and measured the overall efficiency of amplification of Shewanella DNA using a mixture of equal proportions of genomic DNA from ten strains of Shewanella (Fig. 6). The optimal range for quantifying genomic DNA as a function of the qPCR's cycle threshold $(C t)$ results was $10^{-8}-1 \mu \mathrm{g} / \mathrm{ml}$, with a $C t$ ranging between 17 and 40 . At lower template concentrations the variance of $C t$ became too large to allow confident DNA quantification. With an appropriate number of replicates such results can be used to determine errors when quantifying DNA using specific combinations of phylogroup, primers and PCR conditions.

Last but not least, the logarithmic nature of the PCR amplification makes the number of PCR cycles influence the relative fluorescence of the different peaks. Based on the results presented above we have established that obtaining consistent results in ARISA-IGDF requires rigorous and stringent PCR conditions; for the construction of our Shewanella library we used no formamide, 35 PCR cycles, $60{ }^{\circ} \mathrm{C}$ annealing temperature, made amplifications in independent triplicates and fingerprinted the PCR products in two dilutions (1:1 and $1: 7)$.

\subsection{Constructing fingerprints}

In ARISA the electrophoretic bands represent polymers and thus, in the ideal case, averages can be rounded to closest integer values (Hewson and Fuhrman, 2006). However, in most electropherograms peak position precision remains hard to achieve, an incertitude that increases significantly above $500 \mathrm{bp}$. Mistakes in assigning integer values to DNA amplicons, and thus in phylotype identification, made in early steps of analysis have large consequences on fingerprint comparisons, and are very time consuming to correct afterward. In the $82-500$ bp range this problem can be addressed by increasing the number of replicates and by using ladders with denser peaks; yet, this approach becomes cost prohibitive, especially for large DNA fragments where the intrinsic errors of the method are large. We chose using curve

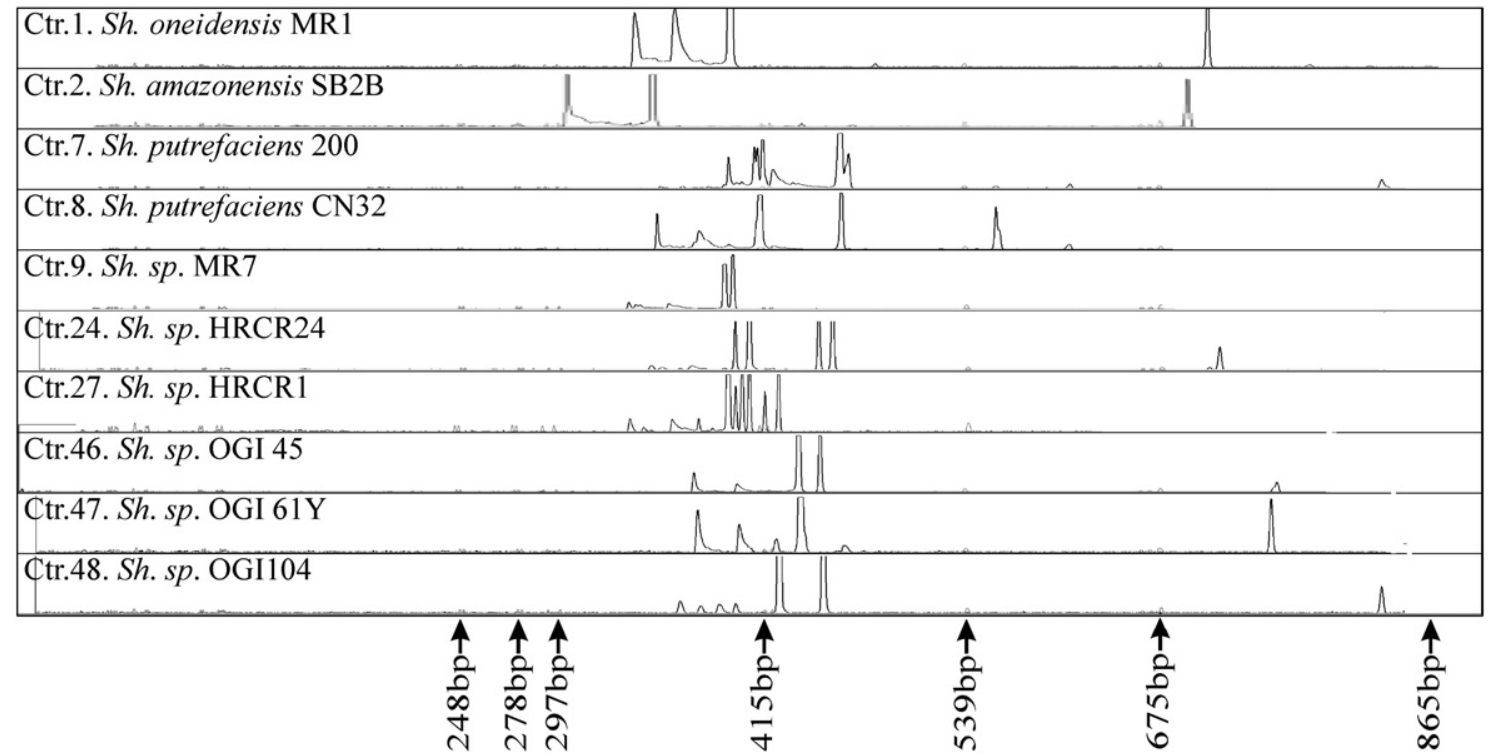



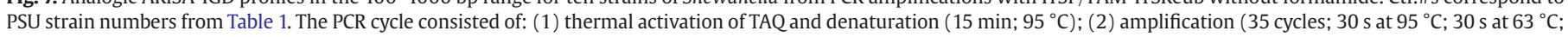
and $20 \mathrm{~s}$ at $\left.72{ }^{\circ} \mathrm{C}\right)$; and (3) final extension $\left(45 \mathrm{~min} ; 72{ }^{\circ} \mathrm{C}\right)$. Numbers on the $x$ axis represent the positions of selected peaks from the ladder. 
Table 2

Digital fingerprint of Shewanella baltica OS155 obtained with the primers ITSF/ITSReub.

\begin{tabular}{lllll}
\hline $\begin{array}{l}\text { Peak } \\
\text { number }\end{array}$ & $\begin{array}{l}\text { Average peak } \\
\text { size (bp) }\end{array}$ & $\begin{array}{l}\text { SD of peak } \\
\text { size (bp) }\end{array}$ & $\begin{array}{l}\text { Mean peak } \\
\text { abundance }(\%)\end{array}$ & $\begin{array}{l}\text { SD of peak } \\
\text { abundance }(\%)\end{array}$ \\
\hline 1 & 438.743 & 0.539 & 0.212 & 0.206 \\
2 & 163.423 & 0.330 & 0.214 & 0.206 \\
3 & 318.715 & 0.448 & 0.219 & 0.206 \\
4 & 143.603 & 0.315 & 0.415 & 0.206 \\
5 & 426.527 & 0.529 & 0.454 & 0.206 \\
6 & 524.339 & 0.604 & 0.529 & 0.207 \\
7 & 863.019 & 0.860 & 0.608 & 0.207 \\
8 & 774.529 & 0.793 & 0.661 & 0.207 \\
9 & 387.718 & 0.500 & 1.064 & 0.207 \\
10 & 772.015 & 0.791 & 1.404 & 0.207 \\
11 & 406.322 & 0.514 & 6.333 & 0.211 \\
12 & 375.817 & 0.491 & 7.734 & 0.212 \\
13 & 796.581 & 0.810 & 8.221 & 0.212 \\
14 & 447.056 & 0.545 & 11.091 & 0.215 \\
15 & 408.696 & 0.516 & 11.976 & 0.215 \\
16 & 377.369 & 0.492 & 12.935 & 0.216 \\
17 & 445.937 & 0.544 & 13.345 & 0.216 \\
18 & 448.300 & 0.546 & 22.583 & 0.223 \\
\hline
\end{tabular}

based estimates of peak sizes and variances obtained from three or more replicates and not assigning integer values to the different DNA fragments. Because in some applications only one or two electropherograms may be practical, or because when comparing peaks between two different electropherograms intrinsic values variance are not available, we used the errors in interpreting peak as a function of fragment size (Eqs. (1) and (2)) to obtain variance estimates.

A systemic difficulty in this analysis was the asymmetry of peaks (i.e. peak shoulders), which can be revealed based on differences between mean and median within the fluorescence data distribution for each peak. Measuring peak height rather than peak area will lower the quantitative determination of fragment abundance, but will help limit the contribution of the surrounding peaks. One approach we have verified to correct peak asymmetry (i.e. peak fronting and peak tailing), was to use peak-deconvolution algorithms to deconstruct asymmetric peaks into a set of smaller peaks. Peak deconvolution is a legitimate approach for analyzing polymers that are different by discrete size intervals, and has the benefit of producing normal peak distribution, thus allowing parametric analysis. Though peak deconvolution is time consuming and impractical in large scale fingerprinting, for very small data sets it may help resolve some of the partly overlapping peaks. We discourage this analysis in ARISA-IGDF, because in this method peak variability and peak asymmetry may be produced by many hard to define and reproduce artifacts, and will result in a number of questionable peaks.

Analogical fingerprints are graphs showing peak abundances relative to fragment sizes. Obtaining them was facilitated by prior description of "Shewanella-specific" primers, and by the sufficiently numerous and variable ITS amplicons in the $82-1000$ bp range for this phylogroup. Some strains of Shewanella have a couple of ITS peaks that are $>1000$ bp large (Fig. 1), and in some phylogroups (such as Rhizobia) ITSs fragments larger than $1 \mathrm{~kb}$ are frequent. Such large fragments are more difficult to measure because the electrophoretic separation becomes too long and errors increase significantly above $1000 \mathrm{bp}$. If ARISA-IGDF is restricted to appropriate phylogroups $(\geq 3$ peaks within the 82-1000 bp range) sufficiently high confidence in strain identification can be obtained (see below). Examples of analogical Shewanella ARISA-IGD fingerprints illustrating the diversity of this phylogroup and the phylogenetic resolution of this method are given in Fig. 7.

Digitizing ARISA-IGD fingerprints has a number of benefits: (1) facilitates applying a variety of statistical procedures; (2) makes data compatible with other libraries created under comparable conditions; (3) facilitates comparisons with in silico ARISA-IGD fingerprints predicted from genomic analysis; and (4) will help in developing software-based screening of fingerprint libraries. One of our goals was to establish a numerical convention that will help formulate a minimalist set of data for digital ARISA-IGD fingerprinting. The size of different ARISA fragments was determined using the GeneScan v2.5 software and validated with Peak Scanner v1.0. We eliminated some of the peaks using the cutoff criteria given in Materials and methods. As different peaks vary in fluorescence considerably, in most samples determining peak abundances required combining data from electropherograms obtained from different dilutions, and remains semiquantitative. We used Eqs. (1) and (2) to assign $S D$ to individual peaks, and cross-compared all peaks from different electropherograms of independent replicates of the same strain (as shown in Materials and methods) to identify similar peaks. The peaks validated for each strain were used to construct a digital fingerprint; one example of such fingerprint is given in Table 2 .

We analyzed digital ARISA-IGD fingerprints of 44 Shewanella strains (Table 1) using two pairs of primers (ITSF/ITSReub and Shew1259F/ITSReub) and the following PCR conditions: $0.34 \mathrm{mg}$ template DNA ml ${ }^{-1}$ PCR reaction; denaturation (15 min; $95{ }^{\circ} \mathrm{C}$ ); 35 cycles (30 s at $94{ }^{\circ} \mathrm{C} ; 30 \mathrm{~s}$ at $60{ }^{\circ} \mathrm{C} ; 1 \mathrm{~min}$ at $72{ }^{\circ} \mathrm{C}$ ); and final extension $\left(45 \mathrm{~min}\right.$ at $\left.72{ }^{\circ} \mathrm{C}\right)$. Differences between the abundances of some ARISA-IGD peaks were very large (e.g. $\geq 50: 1)$; hence, in most cases peak abundance ratios could not be established from a single dilution. Some peaks were too large and saturated the detector (in undiluted 1:1 samples); others were too small to be identified in diluted samples ( $\geq 10: 1$ dilution). For each phylotype and for each pair of primers obtaining ARISA-IGD fingerprints requires six sequencer readings (i.e. three independent PCR amplifications and two 1:7 dilutions for electropherograms).

\subsection{Confidence in phylotype identification}

Confidence in identification using ARISA-IGDF depends on parameters such as: the number of peaks validated for each phylotype; the maximum number of peaks distinguishable in a electropherogram

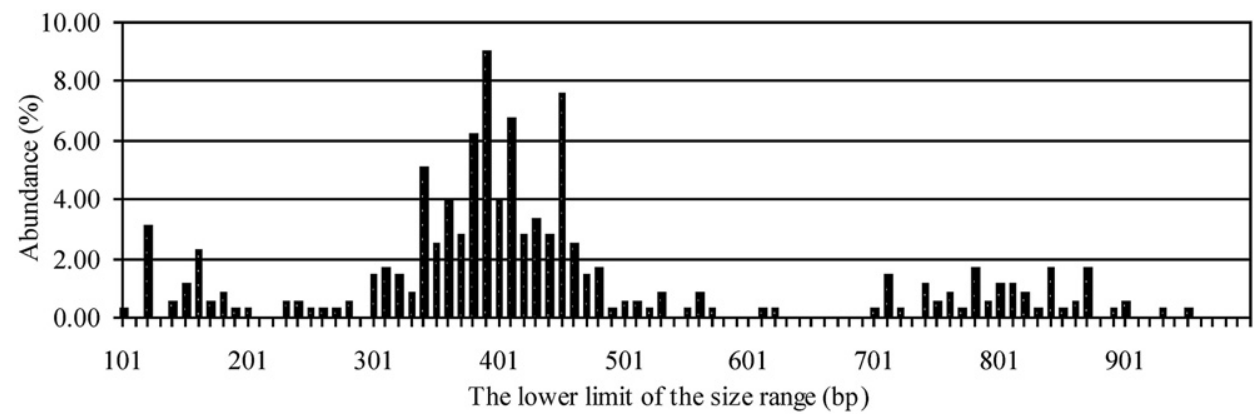

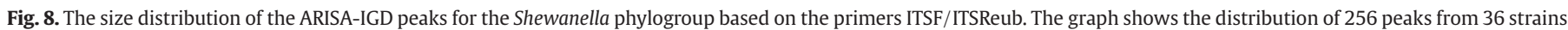
For this analysis $10 \mathrm{bp}$ size ranges were used and we calculated the $p_{i}$ values for each size range. 
and the peak position variability; the total number of phylotypes that can be amplified by a specific set of primers and under specific PCR conditions; the diversity of peak sizes for the phylotypes belonging to a certain phylogroup; and the relative abundance of peaks in each electropherogram. In the simplest case, if the $S D$ of the fragment size is $<1$ bp (Eq. (1)), up to $\sim 300$ distinct peaks may be possibly discriminated in the $82-1000$ bp range, with $~ 97 \%$ confidence (i.e. non-overlapping range for the average $+/-1 S D$ between two adjacent peaks). The probability of an incorrect identification, i.e. the probability for two phylotypes to share a similar number $(n)$ of ARISA bands of similar length and by chance alone is:

$p=1 / 300^{n}$

For evolutionary reasons the distribution of the ARISA-IGDF amplicons within a phylogroup is not even across the $82-1000$ bp size range (Fig. 8). This distribution will probably vary significantly among different phylogroups and with different primers. After analyzing fingerprints of 36 strains of Shewanella from Table 1 we divided this range in 10 bp size intervals, accounted all peaks and calculated the probability for a peak to be present in each specific size interval. Using these $p_{i}$ values the probability of an incorrect identification becomes:

$p(n)=\frac{1}{\prod_{i=1}^{m} i p_{i}}$

where:

$n \quad$ the total number of validated peaks of a phylotype;

$m \quad$ the total number of size range intervals;

$p_{i} \quad$ the probability for a peak to belong to a specific size range.

Using Eqs. ( 3 ) and (4) it can be shown that if $\geq 4$ ARISA-IGDF peaks are present (belonging to four different size ranges), the chance for two different phylotypes of Shewanella to share similar patterns is $\sim 1$ / $300^{4}$ (i.e. one in $8.1 \cdot 10^{9}$ ). No estimates exist regarding the total number of Shewanella phylotypes in nature, or the number of microbes that amplify with these primers at $60{ }^{\circ} \mathrm{C}$; but it is highly unlikely for this number to be anywhere near $10^{9}$. Hence, phylotypes having dissimilar ARISA-IGDF patterns consisting of $\geq 4$ peaks in the 82-1000 bp range can be confidently identified by this method.

When two microbial clones produce amplicons of similar sizes, verifying that they have identical ARISA-IGD fingerprints is helped by ranking peaks based on relative abundances. Fig. 9 gives an example of graphical representation of peak relative abundances. When using relative abundances to compare fingerprints of different strains we recommend using Shannon's index $\left(H^{\prime}\right)$ rather than Simpson's index

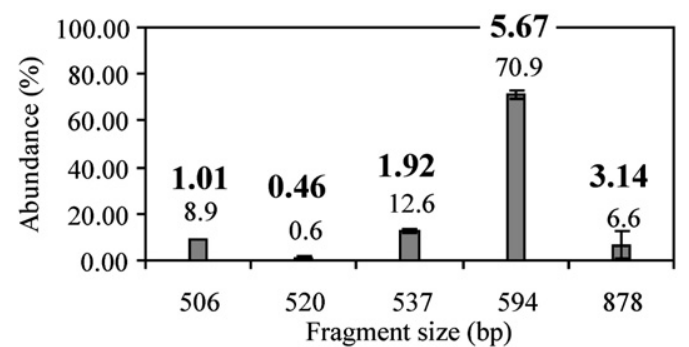

Fig. 9. Relative abundances of five amplicons from an ARISA-IGD profile of $S$ amazonensis SB2B based five independent replicates and using the primers ITSF/ ITSReub. The non-bold font numbers in the graph are the relative abundance of each peak relative to the total fluorescence of all peaks. Error bars are $1 S D$ of relative abundances; the $S D$ values are given in bold font.
(D), which would introduce more biases due to the most fluorescent peaks.

\section{Discussions}

We explored the capacity of ARISA-IGDF to distinguish microorganisms from a target phylogroup by analyzing 44 strains from the genus Shewanella. The stringency of primer/phylogroup recognition improved by increasing the annealing temperature and by adding $1.5 \%$ formamide to the PCR mixture. Formamide had larger inhibition outside the target phylogroup, produced small changes in fingerprint patterns (e.g. some minor peaks were harder to identify and required less stringent cutoffs to reveal), inhibited ( 100 times) the amplification of the target phylogroup (Shewanella) and decreased the annealing temperature by $\sim 3-4{ }^{\circ} \mathrm{C}$. The number of PCR cycles was shown to influence the relative abundance of different amplicons (Daffonchio et al., 2003) and is a key requirement for the reproducibility of this method. Increasing the duration of final extension $\left(72{ }^{\circ} \mathrm{C}\right)$ to $\geq 30 \mathrm{~min}$ eliminated false peaks and made fingerprints more reproducible. High confidence in identification $\left(\sim 8 \cdot 10^{-9}\right)$ can be obtained when using phylotypes with $\geq 4$ distinct ARISA-IGD amplicons in the 82-1000 bp range.

ARISA-IGDF retains a couple of limitations. Amplification of DNA HRCR-8 and E. coli which do not belong to the Shewanella phylogroup occurred with the primers Shew1259F/ITSReub (Shew1259F is supposed to be Shewanella-specific). In such instances using two primers that are group-specific, and increasing the stringency of the PCR conditions by increasing the annealing temperature and adding $1.5 \%$ formamide is recommended. Even if 16S rRNA gene sequence information is available, patterns obtained with the Shew1259F/ITSReub primers can only be useful to a limited extent to predict patterns obtained with ITSF/ITSReub primers, and very little for fragment abundance analysis. This is a primarily a mispriming problem and may be caused by minute sequence differences in the priming areas, differences in optimal annealing conditions between primers, and the presence of the 5'-dye. ARISA-IGD fingerprinting is warped toward culturable microbes, while most species of microbes cannot be isolated in pure cultures. Unresolved peaks from complex samples will be difficult to assign without sequencing the flanking $16 \mathrm{~S}$ rRNA genes as well. Even if good group-specific primers are available, the efficiency of amplification of different strains within a target phylogroup may still remain uneven, and the fingerprinting of microbes with 1-2 ITSs per genome (such as: Sulfolobus, Magnetospirillum, and Magnetococcus) will require ARISA/ tRFLP combinations. More work is needed to compare the performance of ARISA-IGDF with other methods of molecular fingerprinting of high (SSCP, ITS-HHP, Multi Locus Sequence Typing, LH-PCR, Rep-PCR and RAPD) and low phylogenetic resolution (DGGE, TGGE and tRFLP).

\section{Conclusions}

We propose that ARISA-IGDF is a low cost high resolution approach to identify the presence of specific microbes from phylogroups of culturable microorganisms for which group-specific ARISA primers are available. If future work can verify the predictability of ARISA-IGD fingerprints from in silico analysis of genomic data, (and also because once a fingerprint was established there is little need for future sequencing), ARISA-IGDF can be used to complement metagenomic studies in mixed cultures and low diversity microcosms. This method can be applied to monitor the evolution of diversity and the spatial distribution and dynamics of different microorganisms in a little complex community. Other potential applications of ARISA-IGDF include: tracking specific target microbes; identifying sources of microbial contamination; identifying contamination in axenic cultures and routine screening of libraries of microbial cultures. 


\section{Acknowledgements}

This work was supported by a research grant from a NASA Astrobiology Program (NNH07ZDA001N-EXOB) and by a 2008 Portland State University Faculty Enhancement Grant. We thank Dr. Mitch Cruzan and Trieste Dobberstein (PSU) for support with using the ABI 310 sequencer, Dr. Niles Lehman (PSU) for access to the Typhoon gel reader, Dr. James K. Fredrickson (PNNL) for Shewanella strains from the PNNL culture collection, Dr. Ken Stedman for access to the gradient PCR instrument and Dr. Kenneth Nealson, Dr. Ana Obratsova and Shana Rapoport for strains from the USC culture collection.

\section{References}

Acinas, S.G., Anton, J., Rodriguez-Valera, F., 1999. Diversity of free-living and attached bacteria in offshore western Mediterranean waters as depicted by analysis of genes encoding 16S rRNA. Appl. Environ. Microbiol. 65, 514-522.

Bergamo, R.F., Novo, M.T.M., Veríssimo, R.V., Paulino, L.C., Stoppe, N.C., Sato, M.I.Z. Manfio, G.P., Prado Jr., P.I., Garcia, O., Ottoboni, L.M.M., 2004. Differentiation of Acidithiobacillus ferrooxidans and A. thiooxidans strains based on 16S-23S rDNA spacer polymorphism analysis. Res. Microbiol. 155, 559-567.

Cardinale, M., Brusetti, L.P., Quatrini, S., Borin, A.M., Puglia, A., Rizzi, E., Zanardini, C. Sorlini, C., Corselli, B., Daffonchio, D., 2004. Comparison of different primer sets for use in automated ribosomal intergenic spacer analysis of complex bacterial communities. Appl. Environ. Microbiol. 70, 6147-6156.

Cook, A.E., Meyers, P.R., 2003. Rapid identification of filamentous actinomycetes to the genus level using genus-specific 16S rRNA gene restriction fragment patterns. Int. J. Syst. Evol. Microbiol. 53, 1907-1915.

Daffonchio, D., Cherif, A., Brusetti, L., Rizzi, A., Mora, D., Boudabous, A., Borin, S., 2003. Nature of polymorphisms in 16S-23S rRNA gene intergenic transcribed spacer fingerprinting of Bacillus and related genera. Appl. Environ. Microbiol. 69, 5128-5137.

Emerson, D., Moyer, C., 1997. Isolation and characterization of novel iron-oxidizing bacteria that grow at circumneutral pH. Appl. Environ. Microbiol. 63, 4784-4792.

Fisher, M.M., Triplett, E.W., 1999. Automated approach for ribosomal intergenic spacer analysis of microbial diversity and its application to freshwater bacterial communities. Appl. Environ. Microbiol. 65, 4630-4636.

Fredrickson, J.K., Romine, M.F., Beliaev, A.S., Auchtung, J.F., Driscoll, M.E., Gardner, T.S., 2008. Towards environmental systems biology of Shewanella. Nature 6, 592-603.

Garcia-Martinez, J., Acinas, S.G., Anton, A.I., Rodriguez-Valera, F., 1999. Use of the 16S$23 \mathrm{~S}$ ribosomal genes spacer region in studies of prokaryotic diversity. J. Microb. Methods 36, 55-64.

Grassi, M., Volpe, E., Colizzi, V., Marian, F., 2006. An improved, real-time PCR assay for the detection of GC-rich and low abundance templates of Mycobacterium tuberculosis. J. Microb. Methods 64, 406-410.
Heuer, H., Krsek, M., Baker, P., Smalla, K., Wellington, E.M.H., 1997. Analysis of actinomycete communities by specific amplification of genes encoding 16S rRNA and gel-electrophoretic separation in denaturing gradients. Appl. Environ. Microbiol. 63, 3233-3241.

Hewson, I., Fuhrman, J.A., 2006. Improved strategy for comparing microbial assemblage fingerprints. Microb. Ecol. 51, 147-153.

Hewson, I., Vargo, G.A., Fuhrman, J.A., 2003. Bacterial diversity in shallow oligotrophic marine benthos and overlying waters: effects of virus infection, containment, and nutrient enrichment. Microb. Ecol. 46, 322-336.

Jensen, M.A., Webster, J.A., Straus, N., 1993. Rapid identification of bacteria on the basis of polymerase chain reaction-amplified ribosomal DNA spacer polymorphisms. Appl. Environ. Microbiol. 59, 945-952.

Loisel, P., Harmand, J., Zemb, O., Latrille, E., Lobry, C., Delgenes, J.P., Godon, J.J., 2006 Denaturing gradient electrophoresis (DGE) and single-strand conformation polymorphism (SSCP) molecular fingerprintings revisited by simulation and used as a tool to measure microbial diversity. Environ. Microbiol. 8, 720-731.

Mazza, P., Monciardini, P., Cavaletti, L., Sosio, M., Donadio, S., 2003. Diversity of Actinoplanes and related genera isolated from an Italian soil. Microb. Ecol. 45, 362-372.

Ni, Y.Q., Yang, Y., Bao, J.T., He, K.Y., Li, H.Y., 2007. Inter- and intraspecific genomic variability of the $16 \mathrm{~S}-23 \mathrm{~S}$ intergenic spacer regions (ISR) in representatives of Acidithiobacillus thiooxidans and Acidithiobacillus ferrooxidans. FEMS Microbiol. Lett. 270, 58-66.

Offre, P., Pivato, B., Siblot, S., Gamalero, E., Corberand, T., Lemanceau, P., Mougel, C., 2007. Identification of bacterial groups preferentially associated with mycorrhizal roots of Medicago truncatula. Appl. Environ. Microbiol. 73, 913-921.

Ranjard, L., Lejon, D.P.H., Mougel, C., Schehrer, L., Merdinoglu, D., Chaussod, R., 2003. Sampling strategy in molecular microbial ecology: influence of soil sample size on DNA fingerprinting analysis of fungal and bacterial communities. Environ. Microbiol. 5, 1111-1120.

Robleto, E.A., Borneman, J., Triplett, E.W., 1998. Effects of bacterial antibiotic production on rhizosphere microbial communities from a culture-independent perspective. Appl. Environ. Microbiol. 64, 5020-5022.

Sarkar, G., Kapelner, S., Sommer, S.S., 1990. Formamide can dramatically improve the specificity of PCR. Nucleic Acids Res. 18, 7465.

Siering, P.L., Ghiorse, W.C., 1996. Phylogeny of the Sphaerotilus-Leptothrix group inferred from morphological comparisons, genomic fingerprinting, and 16S ribosomal DNA sequence analyses. Int. J. Syst. Bacteriol. 46, 173-182.

Todorova, S.G., Costello, A.M., 2006. Design of Shewanella-specific 16S rRNA primers and application to analysis of Shewanella in a minerotrophic wetland. Environ. Microbiol. 8, 426-432.

Torzilli, A.P., Sikaroodi, M., Chalkley, D., Gillevet, P.M., 2006. A comparison of funga communities from four salt marsh plants using automated ribosomal intergenic spacer analysis (ARISA). Mycologia 98, 690-698.

Zhang, W., Albert, B., 1996. Direct PCR sequencing with denaturants (formamide). Methods Mol. Biol. 65, 137-147. 